\title{
Temporal pattern of the mechanoluminescent lighting from impact-loaded $\mathrm{ZnS}$ ceramics
}

\author{
AleXANDre ChMel $^{1, *}$, AnAtolij Dunaev ${ }^{2}$, IgOr ShCHerbakov $^{1}$ \\ ${ }^{1}$ Ioffe Institute, 194021 St. Petersburg, Russia \\ ${ }^{2}$ Vavilov State Optical Institute, 192171 St. Petersburg, Russia
}

\begin{abstract}
Mechanoluminescence (ML) from deformed ductile materials is caused by sliding of charged dislocations. The main source of the ML in loaded brittle solids is the interatomic bond breakage. In this work, the ML from impact damaged ductile $\mathrm{ZnS}$ ceramics was studied. It was revealed that the time series of ML pulses exhibited two well-separated peaks. A two-stage ML excitation of this kind in $\mathrm{ZnS}$ ceramics was observed when the applied load exceeded an ultimate plastic deformation. The positions of each peak along the time axis were found to be dependent in different ways on physical and mechanical properties of crystallites that constituted the given ceramics. The investigated ceramics were produced by four different technological methods. The obtained samples differing in the dimensions of grains and separation of grain boundaries were tested. The statistical analysis of a temporal pattern of the ML lighting showed that the mechanisms of excitation of the two peaks were not the same. The first ML peak was assigned to the plastic deformation preceding the ceramics cracking. The second peak originated from the interatomic bond breakage in nucleated and growing cracks.
\end{abstract}

Keywords: ZnS ceramics; impact damage; mechanoluminescence; time series

\section{Introduction}

IR optical material $\mathrm{ZnS}$ is widely used in luminescent sensors of dynamic loading in aircraft industry [1] as well as in elements of solar cells [2] and serves as protective windows in mobile forward-looking devices [3, 4]. As a rule, outdoor optical elements are the elements subjected to hit of dust particles and atmospheric precipitations $[5,6]$. Therefore, the mechanisms of their deformation and fracture under the impact loading have drawn attention of specialists over a protracted period [7-10]. Depending on specificity of application, the $\mathrm{ZnS}$ compound could be used in the form of single crystals [11], ceramics [12], or films [13].

In most bulk applications, zinc sulfide in the form of ceramics is the preferred choice as compared to its single-crystal counterparts due to easy changing of geometrical shape, isotropy of mechanical properties, and its lower cost.

\footnotetext{
*E-mail: chmel@mail.ioffe.ru
}

The presented investigation of the ceramics response to the impact deformation and fracture was carried out by the mechanoluminescence (ML) method. The ML effect is a special case of the triboluminescence phenomenon; however, in recent times, the term "triboluminescence" is commonly referred to the light generation through rubbing of a material, while the photon emission from deformed or fractured solids is more frequently identified as "mechanoluminescence". In ductile materials, such as alkali halide crystals and II-VI semiconductors, the ML occurs when a solid undergoes mechanical deformation. The presence of electric field around the moving dislocations causes the band bending [7], and, correspondingly, the tunneling of electrons from filled traps to the conduction band with consequent light emitting electron-hole recombination [9].

$\mathrm{ZnS}$ is made up of covalent bonds with an ionic character [14]. The ML lighting from fractured brittle covalent and ionic-covalent solids is caused, mainly, by multiple interatomic bond breaking in growing cracks, which change locally the electronic configuration. This process is accompanied 
with the light emission from the electronic structures reconfigured during the covalent bond breaking [15].

To our best knowledge, the ML effect in undoped $\mathrm{ZnS}$ and other II-VI semiconductors has been studied in detail only for monocrystalline samples. Meanwhile, various $\mathrm{ZnS}$ mechanoluminescent sensors and impact protection shields have been constructed mainly from ceramics. The heterogeneity inherent to ceramic materials governs the mechanical response to the external loading [16]. In this research, the impact damage of ceramic $\mathrm{ZnS}$ plates has been studied. A set of samples of ceramics manufactured by various methods, which had quite different grain size and properties of intergranular boundaries was tested.

\section{Experimental}

\subsection{Samples}

A set of self-obtained and commercial ceramics prepared using various techniques was tested:

A. Hot pressing (HP) of fine-grained $(30-50 \mu \mathrm{m})$ crystallites in vacuum $1.33 \mathrm{~Pa}$ at $1200{ }^{\circ} \mathrm{C}$ under uniaxial pressure of $200 \mathrm{MPa}$ during $30 \mathrm{~min}$.

B. Physical vapor deposition (PVD) in vacuum of $0.13 \mathrm{~Pa}$ at the temperature $1000{ }^{\circ} \mathrm{C}$ and the rate of raw vaporization of $0.5 \mathrm{~mm} / \mathrm{h}$.

C. Chemical vapor deposition (CVD) under pressure of $13 \mathrm{GPa}$ in the gas mixture composed of ( $\mathrm{Ar}$ $+\mathrm{H}_{2} \mathrm{~S}+\mathrm{Zn} \uparrow$ ).

D. Additional isostatic hot pressing (HIP) procedure of the CVD-synthesized material. This treatment does not cause any recrystallization but reduces substantially intergranular boundaries.

Some characteristics of the applied methods are given in Table 1.

Hereafter, the samples are denoted by upper indexes HP, PVD, CVD, CVD+HIP (i.e. $\mathrm{ZnS}^{\mathrm{HP}}$, $\mathrm{ZnS}^{\mathrm{PVD}}$, etc.). The samples were shaped into discs of min. 20 to $30 \mathrm{~mm}$ in diameter and $\mathrm{min}$. 1 to $2 \mathrm{~mm}$ in thickness. The specimens grown by CVD and PVD methods were cut normally to the growth direction.
Table 1. Some characteristics of tested $\mathrm{ZnS}$ ceramics.

\begin{tabular}{lccc}
\hline Method & $\begin{array}{c}\text { Max. processing } \\
\text { temperature } \\
{\left[{ }^{\circ} \mathrm{C}\right]}\end{array}$ & $\begin{array}{c}\text { Average } \\
\text { grain size } \\
{[\mu \mathrm{m}]}\end{array}$ & $\begin{array}{c}\text { Growth } \\
\text { rate } \\
{[\mu \mathrm{m} / \mathrm{h}]}\end{array}$ \\
\hline \hline HP & 950 & $\sim 1$ & 800 \\
PVD & 1000 & $>200$ & 400 \\
CVD & 750 & $\sim 10$ & 70 \\
CVD+HIP & 980 & $>200$ & 100 \\
\hline
\end{tabular}

\subsection{Experimental setup}

A schematic diagram of the experimental setup is depicted in Fig. 1. The samples were placed on a massive metal support covered with a grease layer. A surface damage was produced by a pointed striker positioned on the upper face of the sample, on which a $100 \mathrm{~g}$ weight dropped. The data acquisition system was triggered in the moment of contact between the weight and the striker.

The ML radiation from the samples was collected with a quartz lens and directed onto a photomultiplier FEU136. The single-electron pulse duration of the FEU136 was 8 ns. The characteristic time of the resistance-capacitance network (cables + photomultiplier) was 5 ns. An analogueto-digital converter ASK-3106 provided a dynamic range of $2 \mathrm{mV}$ to $10 \mathrm{~V}(70 \mathrm{~dB})$ in the time range of $10 \mathrm{~ns}$ to $100 \mathrm{~s}$. The converted (digital) ML signals were stored in a PC. The duration of all recorded time series was $0.4 \mathrm{~ms}$.

\subsection{ML amplitudes}

The time series of the ML pulses from the impact damaged $\mathrm{ZnS}$ ceramics manufactured by various methods and, correspondingly, characterized by various degree of heterogeneity are depicted in Fig. 2. One can see that the ML excitation manifests itself by two peaks separated by a relatively quiet period. (Hereafter, the first occurring maximum will be denoted as "peak 1", and the second maximum as "peak 2".) Time delay between a shock and the beginning of lighting was the shortest in the smallest-grained $\mathrm{ZnS}^{\mathrm{HP}}$ ceramics. The ML excitation from the largest-grained $\mathrm{ZnS}{ }^{\mathrm{PVD}}$ ceramics appeared later than that from other samples. The $\mathrm{ZnS}^{\mathrm{CVD}}$ sample exhibited an intermediate 


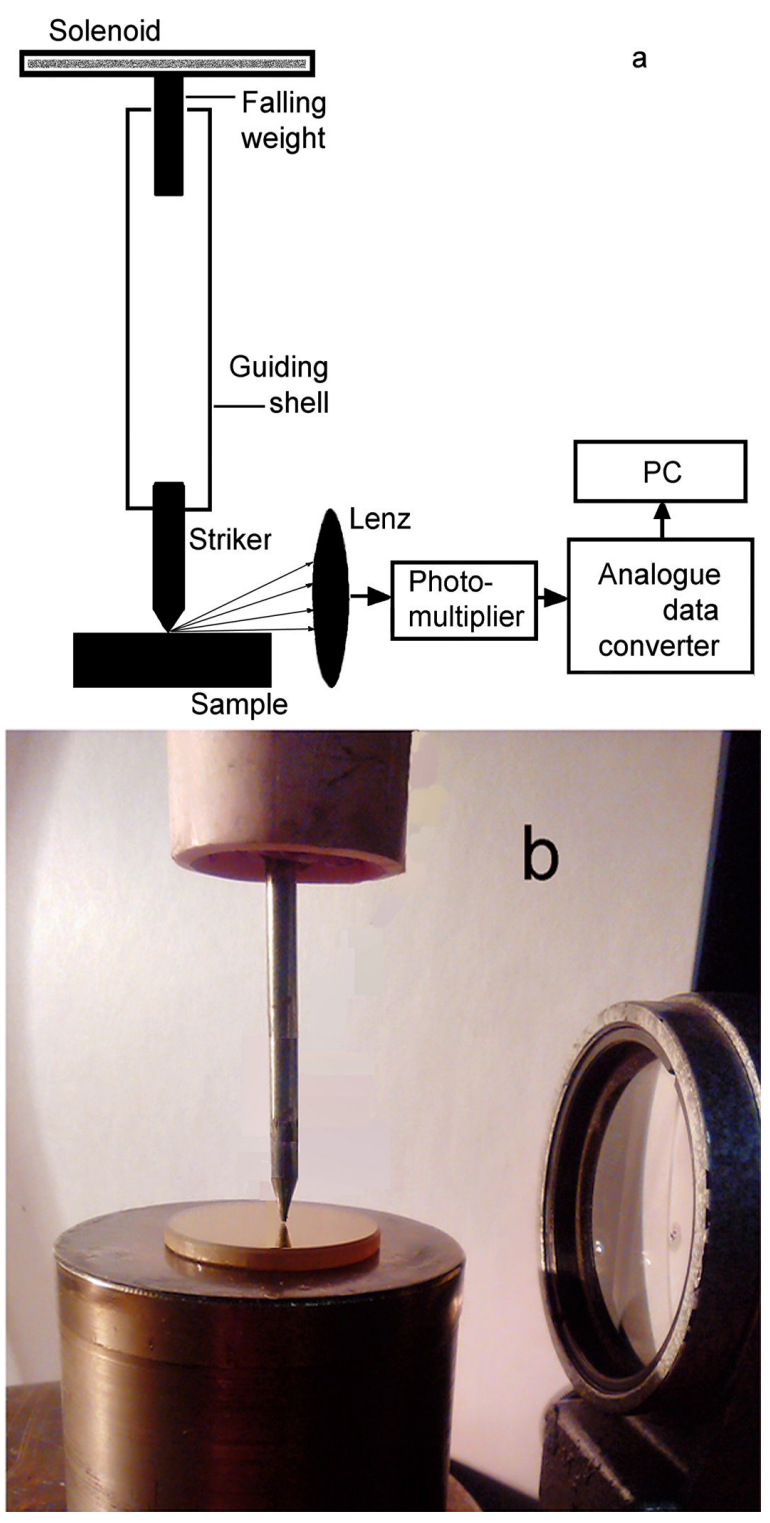

Fig. 1. Schematic diagram and photograph of the experimental setup.

delay, and this delay decreased slightly after the HIP treatment $\left(\mathrm{ZnS} \mathrm{CVD}^{\rightarrow} \mathrm{ZnS}^{\mathrm{CVD}+\mathrm{HIP}}\right)$.

\subsection{Waiting time}

In order to compare statistics of ML generation in the mentioned time series, distributions of the time intervals between subsequent pulses ("waiting times" for next signal) were created in the form of the $\mathrm{N}(\mathrm{t}>\tau)$ of $\tau$ dependences where $\mathrm{N}$ is the number of intervals of duration of $t$ that exceeded

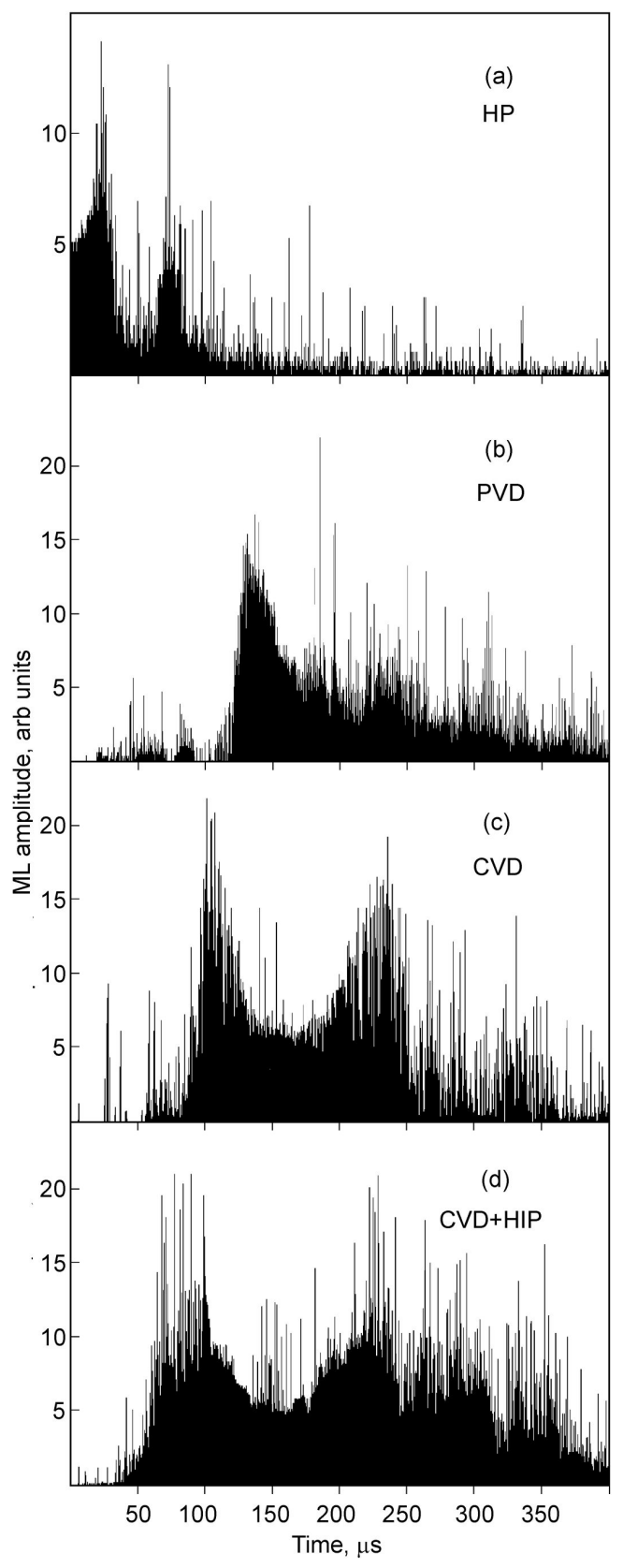

Fig. 2. Time series of ML pulses from impactloaded $\mathrm{ZnS}^{\mathrm{HP}}$ (a), $\mathrm{ZnS}^{\mathrm{PVD}}$ (b), $\mathrm{Zn}^{\mathrm{CVD}}$, and $\mathrm{ZnS}^{\mathrm{CVD}+\mathrm{HIP}}$ (d) ceramics.

an arbitrary value of $\tau$. Fig. 3 shows these dependences plotted in double-logarithmic coordinates. One can see that each time distribution contains a log-linear portion corresponding to the relation:

$$
\log N(t>\tau) \propto-\gamma \log \tau
$$





Fig. 3. Waiting times distributions in time series of ML pulses from impact loaded $\mathrm{ZnS}^{\mathrm{HP}}$ (a), $\mathrm{ZnS}^{\mathrm{PVD}}$ (b), $\mathrm{Zn}^{\mathrm{CVD}}$ (c), and $\mathrm{ZnS} \mathrm{S}^{\mathrm{CVD}+\mathrm{HIP}}$ (d) ceramics.

which represents the power law dependence:

$$
N(t>\tau) \propto t^{-\gamma}
$$

indicative of the scale-invariant temporal pattern of the ML excitation. In contrast to an exponential (random) time distribution, a power law function is specific for correlated events.

The scale invariance signalizes a constant proportion between "short" and "long" waiting times in all time periods of the ML generation: all fragments of the $\mathrm{N}(\mathrm{t}>\tau)$ of $\tau$ dependence show the same slope, which has been characterized numerically by the parameter $\gamma$. The higher $\gamma$, the smaller part of larger intervals between FL pulses. For example, the slopes of the first-peak-related loglinear dependences were always higher than the slopes corresponding to peak 2.

A deviation from the log-linear behavior for the shortest intervals was caused by the limited time resolution of the experimental setup (10 ns). The log-linear dependences for the "shortest" intervals were also disturbed because of insufficient number of large damages (statistical insignificance).

One can see that the slope of log-linear portions corresponding to peak 1 was higher in all samples than the slope of peak 2 . The slopes were characterized numerically by the value of the parameter $\gamma$ in equation 1 . The $\gamma$-value gives a semi-quantitative estimate of the proportions of "short" and "long" waiting times.

\section{Discussion}

The mechanical behavior of $\mathrm{ZnS}$ ceramics as well as other heterogeneous materials is determined, in many respects, by the grain size and specific properties of intergranular boundaries [16]. The role of heterogeneity manifested itself in the experiments regarding impact loading of $\mathrm{ZnS}$ ceramics. First, each time series included a quiet period prior to the primary ML excitation after the load application (Fig. 2). This "dead zone" might be caused by sliding of crystallites between each other without intrinsic deformation. This period may be regarded as a preliminary stage of damage formation when the crystalline structure remains untouched. Second, the delay of lighting was shorter in small-grained $\mathrm{ZnS}^{\mathrm{HP}}$, while the beginning of ML generation occurred later in $\mathrm{ZnS} \mathrm{S}^{\mathrm{PVD}}$ and $\mathrm{ZnS}{ }^{\mathrm{CVD}}$ ceramics built from larger crystallites. The HIP treatment of the chemically deposited material $\left(\mathrm{ZnS} \mathrm{SVD}^{\mathrm{CV}+\mathrm{HIP}}\right)$ resulted in a certain decrease 
of the "dead zone" due to shortened intergranular sliding cycle in more compacted ceramics.

As fast as the grain sliding diminished, the first ML peak caused by the dislocation motion in crystallites appeared. The intergranular boundaries impeded the dislocation motion and possibility of their travelling from grain to grain, thus strengthening the deformed material [17]. Therefore, the first stage of the ML lighting in small-grained $\mathrm{ZnS}^{\mathrm{HP}}$ sample was restricted by the free path of dislocations inside the crystallites. In $\mathrm{ZnS} \mathrm{CVD}^{\mathrm{CHIP}}$, the role of intergranular boundaries was insignificant.

The primary ML decayed with achieving the ultimate deformation. Further penetration of the striker into the ceramics caused multiple bond breakage accompanied with local reconfiguration of the electronic structure, and a second splash of ML lighting (peak 2). The difference in the mechanisms of ML excitation, which gave rise to either first or second peak was supported by the statistical distribution of interval between ML pulses in the peaks.

Actually, all calculated $\mathrm{N}(\mathrm{t}>\tau)$ of $\tau$ dependences followed a power law. However, the slopes of log-linear plots in the distributions corresponding to peaks 1 differed from those corresponding to peaks 2: the $\gamma$-value was higher at the first stage of ML lighting thus indicating the prevalence of short waiting times during plastic deformation. In other words, unit events of deformation occurring at the nanostructural scale level are less time-consuming than crack nucleation.

\section{Conclusion}

The presented results show that the impact force on $\mathrm{ZnS}$ ceramics is accompanied with mechanoluminescence lighting which proceeds in two stages. The ML excitation is related with the electronic processes occurring either below the ultimate deformation or after the cracking beginning. The first stage is caused by the travelling of charged dislocations to grain boundaries, which manifest themselves as stoppers of dislocations. This effect is known to be increasing yield strength of deformed material. Provided the further external forcing causing the ultimate deformation, the crack nucleation begins with a new cycle of lighting (second stage) due to reconfiguration of the electronic structures in the vicinity of breaking in the lattice continuity in crystallites. The difference in the mechanisms of ML excitation in two subsequent light splashes was confirmed by the statistics of time intervals between ML pulses at two stages of impact-induced damage formation.

\section{References}

[1] Kobakhidze L., Guidry C.J., Hollerman W.A. FONTENOT R.S., IEEE Sensors J., 13 (2013), 3053.

[2] Ummartyotin S., Infahsaeng Y., Renew. Sust. Energ. Rev., 55 (2016), 17.

[3] Klein C.A., Dibenedetto B., Pappis J., Opt. Eng., 25 (1986), 519.

[4] Chaffin J.H.-III, Patent US5242709 A, 1993.

[5] Kennedy C.F., Field J.E., J. Mater. Sci., 35 (2000), 5331.

[6] JILBERT G.H., FIELD J.E., Wear, 243 (2000), 6.

[7] BRedikHin S.I., ShMurak S.Z., JETP, 49 (1979), 520.

[8] Chandra B.P., Chandra V.K., Jha P., Pateria D., BAGHEL R.N., Lumin., 31 (2016), 667.

[9] Tiwari R., Dubey V., Ramrakhiani M., ChanDRA B.P., Lumin., 30 (2015), 883-90.

[10] Robsman V.A., Shikhsaidov M.SH., Phys. Solid State, 30 (1988), 3329.

[11] Saleh M., LYNn K.G., MCCloy J.S., Evaluation of undoped $\mathrm{ZnS}$ single crystal materials for $x$-ray imaging applications, in: ZELINSKI B.J. (Ed.), Window and Dome Technologies and Materials XV, Proc. SPIE 10179, Anaheim, USA, 2017, P. 1017904.

[12] Rozenburg K.G., URruti E.H., Patent USA 2013/0271610 A1.

[13] Zaware R., Wagh B., Mater. Sci.-Poland, 32 (2014), 375.

[14] Pauling L., The nature of the chemical bonds, third edition, Cornell University Press, Ithaca, New York, 1960.

[15] Chmel A., Shcherbakov I., J. Lumin., 153 (2014), 85.

[16] Townsend D., Field J.E., J. Mater. Sci., 25 (1990), 1347.

[17] Pelleg J., Mech. Prop. Mater, Springer Science + Business, Netherlands, Dordrecht, 2013.

Received 2017-09-27

Accepted 2019-04-23 